\title{
Papers
}

\section{Effect of breast feeding in infancy on blood pressure in later life: systematic review and meta-analysis}

\author{
Christopher G Owen, Peter H Whincup, Julie A Gilg, Derek G Cook
}

\begin{abstract}
Objective To determine whether breast feeding in infancy compared with bottle feeding formula milk is associated with lower mean blood pressure at different ages.

Design Systematic review.

Data sources Embase, Medline, and Web of Science databases. Study selection Studies showing the effects of feeding in infancy on blood pressure at different ages.

Data extraction Pooled mean differences in blood pressure between breast fed infants and those bottle fed formula milk, based on random effects models.

Data synthesis The pooled mean difference in systolic blood pressure was $-1.10 \mathrm{~mm} \mathrm{Hg}(95 \%$ confidence interval -1.79 to $-0.42 \mathrm{~mm} \mathrm{Hg}$ ) but with significant heterogeneity between estimates $(\mathrm{P}<0.001)$. The difference was largest in studies of $<300$ participants $(-2.05 \mathrm{~mm} \mathrm{Hg},-3.30$ to $-0.80 \mathrm{~mm} \mathrm{Hg}$ ), intermediate in studies of 300-1000 participants $(1.13 \mathrm{~mm} \mathrm{Hg}$, -2.53 to $0.27 \mathrm{~mm} \mathrm{Hg}$ ), and smallest in studies of $>1000$ participants $(-0.16 \mathrm{~mm} \mathrm{Hg},-0.60$ to $0.28 \mathrm{~mm} \mathrm{Hg}$ ). An Egger test but not Begg test was statistically significant for publication bias. The difference was unaltered by adjustment for current size and was independent of age at measurement of blood pressure and year of birth. Diastolic blood pressure was not significantly related to type of feeding in infancy.

Conclusions Selective publication of small studies with positive findings may have exaggerated claims that breast feeding in infancy reduces systolic blood pressure in later life. The results of larger studies suggest that feeding in infancy has at most a modest effect on blood pressure, which is of limited clinical or public health importance.
\end{abstract}

\section{Introduction}

Blood pressure in adulthood has a strong influence on risk of coronary heart disease and stroke. ${ }^{1}$ It has been postulated that factors operating in early life (in utero and in infancy and childhood), influence the development of blood pressure in adulthood, and that nutrition early in life may programme subsequent blood pressure. ${ }^{2-4}$

The influence of breast feeding on blood pressure is of interest because of the differing composition of breast milk and formula milk, particularly the sodium and fatty acid content. Until the 1980s the sodium content of breast milk in Western countries was much lower than that of formula milk. ${ }^{56}$ Low sodium intake in infancy has been related to lower levels of blood pressure both in the short term and in the long term. ${ }^{78}$ Long chain polyunsaturated fatty acids are present in breast milk but not in formula milk. These play an important part in the vas- cular endothelium and, when given as nutritional supplements, seem to reduce blood pressure in adults and children. ${ }^{9-12}$

Small observational studies suggest that breast feeding may be related to noticeably lower blood pressure in childhood. ${ }^{13-15}$ Similar conclusions were reached by a follow up study of participants in a randomised controlled trial of feeding in preterm infants. ${ }^{16}$ Not all published studies have reported an association. ${ }^{17}$ We performed a systematic review to examine whether there are consistent mean differences in blood pressure between adults who were initially breast fed or bottle fed with formula milk.

\section{Methods}

We searched Embase, Medline, and Web of Science databases for published papers, letters, abstracts, and review articles on the effects of feeding in infancy on blood pressure. Relevant references were identified using a combined text word and MESH or subject heading (for Medline and Embase only) search strategy of infant feeding (breast fed, feed\{s/ing\}, milk; milk, human; bottle fed, feed $\{\mathrm{s} /$ ing $\}$; infant feed $\{\mathrm{s} / \mathrm{ing}\}$, nutrition; formula fed, feed $\{\mathrm{s} / \mathrm{ing}\}$ ) and blood pressure (blood pressure, or hypertension). We restricted our review to studies in humans.

The electronic search (completed March 2003) identified 339 references; a review of the abstracts showed that 35 were potentially relevant and an additional 10 were likely to have relevant data. We excluded 16 of these 45 papers after further review. Thus 29 papers were considered relevant because either the abstract indicated that blood pressure had been measured in different infant feeding groups or the data were provided. ${ }^{12-19} \mathrm{w1}-\mathrm{w} 21$ For all studies we sought the difference in systolic and diastolic blood pressure between those exclusively breast fed and those exclusively bottle fed, adjusted for current age and sex (if appropriate) and for height and body mass index. In studies including different ethnic groups, we sought additional adjustment for ethnicity. ${ }^{13}$ w8 Information was obtained from 14 of the 29 studies directly (with various levels of adjustment), and we approached the investigators of the other studies for further results. Results were obtained for 11 of these studies; we had direct access to two, and we obtained data from the archives of the 1970 British cohort study (10 year olds). ${ }^{17}{ }^{19}$ No responses were elicited for four studies; two were large, well conducted, observational studies, and the others were small $(<100$ participants) and in infants and children (aged 1 and 6, respec-

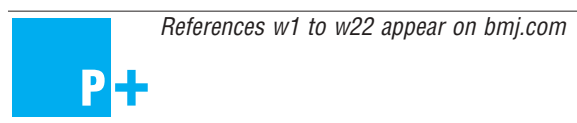


tively). ${ }^{\mathrm{w} 5}$ w6 w8 w13 One reviewer (CGO) completed the search and extracted data from the studies on two occasions. The 25 studies for which data were obtained had information on systolic blood pressure and 22 also had data on diastolic blood pressure. One study gave three estimates, providing 27 estimates of systolic blood pressure and 24 estimates of diastolic blood pressure. ${ }^{\text {w16 }}$

Differences in blood pressure between infant feeding groups were collected on more than one occasion in three studies from the same cohort. ${ }^{16} 18$ w3 w19 w22 In these studies data from the earliest age was used for overall estimates. ${ }^{18}$ w w19 $I n$ one of these studies, with high response rates (at 7 years and 18 years), both estimates were used in the age specific analysis. ${ }^{\mathrm{w} 19}$ Overall, there were 26 estimates of systolic blood pressure and 23 estimates of diastolic blood pressure.

In most studies the feeding groups were separate; in one study the breastfed infants were partly bottle fed and in another study the bottle fed infants were partly breast fed. ${ }^{\text {w3 }}{ }^{15}$ In 10 additional studies we were unable to verify the exclusiveness of infant feeding. Bottle fed infants receiving standard formulas were included in preference to those receiving specially supplemented formulas. Except for one study, we were able to systematically exclude these from the analysis. ${ }^{18}$

\section{Statistical analysis}

In the meta-analysis we used the mean difference in systolic blood pressure between infant feeding groups (breast fed and bottle fed) and the standard error of the difference from individual studies. The META command in STATA was used across studies to test for heterogeneity of the differences in systolic blood pressure between infant feeding groups. Because heterogeneity was noticeable, we produced pooled estimates of the difference in systolic blood pressure using random effects models..$^{20}$ Publication bias was assessed with funnel plots. ${ }^{21}$ Begg and Egger tests were also performed..$^{22}{ }^{23}$ Analyses were carried out after stratifying by study size in three groups chosen a priori: less than 300 participants; 300 to 1000 participants; and more than 1000 participants. The METAREG command was used to explore whether there were differences between age groups based on the mean age of the sample (infants $\leq 1$ year, children $>1$ to 16 years, adolescents and adults $>16$ years), dates of birth (including whether born before or after 1980), the impact of ascertaining infant feeding status in infancy or by parental questionnaire at least three years after birth, and the difference in effect size between studies that reported on the association between infant feeding and blood pressure and those that did not. ${ }^{13}$ w19 The effect of adjustment for current body size (height and body mass index) was also examined in 10 studies (12 observations), with data before and after adjustment.

\section{Assessment of study quality}

Methods for defining study quality in observational studies are less clearly defined than those for experimental studies. We used sensitivity analyses to examine the effects of different methods of ascertaining exposure (particularly contemporary ascertainment or recalled ascertainment) and to examine the effects of excluding groups that used mixed feeding methods. It

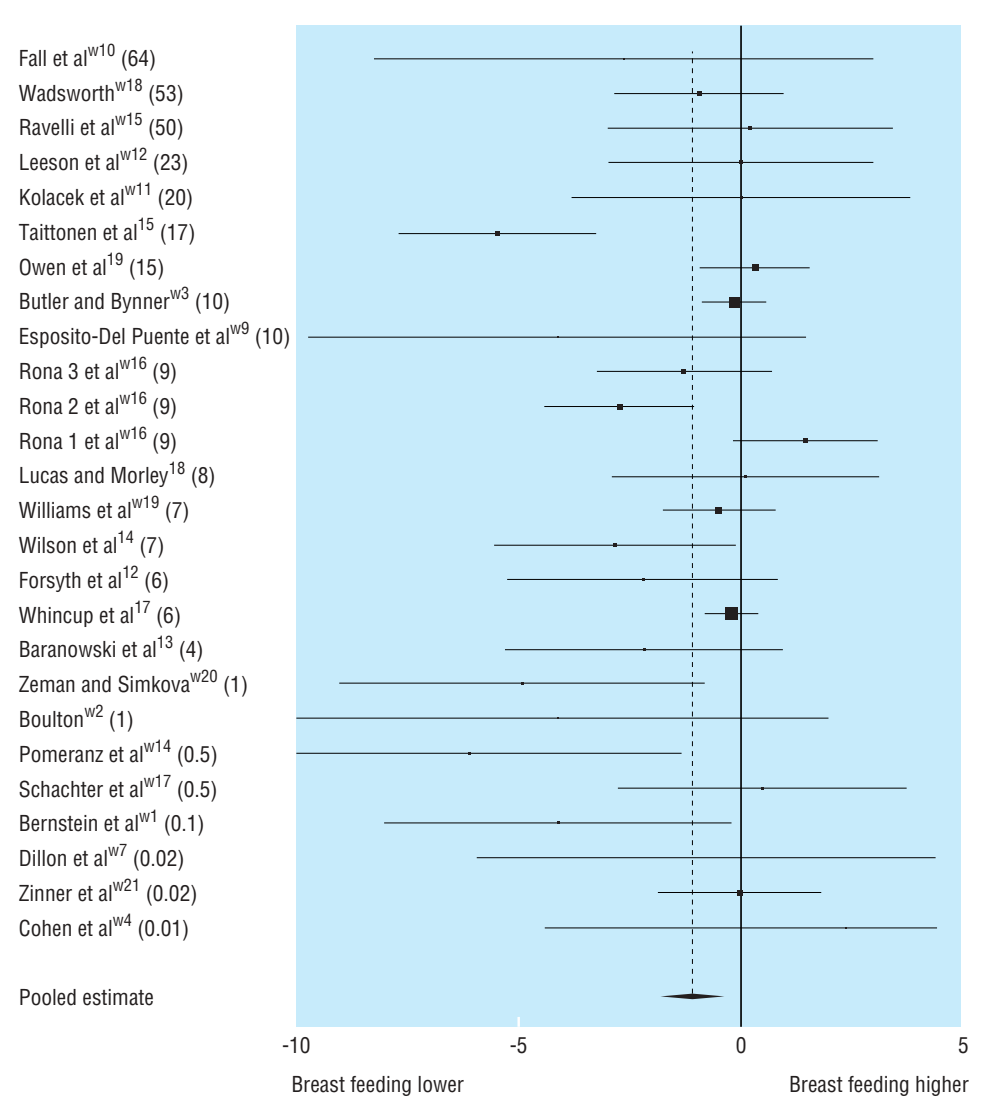

Mean difference in systolic blood pressure $(\mathrm{mm} \mathrm{Hg})$

Fig 1 Mean differences in systolic blood pressure (95\% confidence intervals) between breastfed and bottle fed participants. Box area proportional to inverse of variance, with horizontal lines showing 95\% confidence intervals. y axis in ascending order of mean age (years) at which blood pressure was measured. Dashed vertical line and diamond (95\% confidence interval) is pooled estimate based on a random effects model 


\begin{tabular}{|c|c|c|c|c|c|c|c|c|}
\hline Study & Design & Source & $\begin{array}{l}\text { Source of } \\
\text { information } \\
\text { on feeding }\end{array}$ & $\begin{array}{l}\text { Year } \\
\text { born }\end{array}$ & $\begin{array}{l}\text { Age at } \\
\text { blood pressure } \\
\text { measurement }\end{array}$ & $\begin{array}{l}\text { No breast } \\
\text { fed, } \\
\text { No bottle fed }\end{array}$ & $\begin{array}{l}\text { Formula type, } \\
\text { comments }\end{array}$ & $\begin{array}{l}\text { Exclusive feeding } \\
\text { groups }\end{array}$ \\
\hline Cohen $^{\text {w4 }}$ & CS & $\begin{array}{l}\text { Born at North Central Bronx } \\
\text { Hospital or Newark Israel } \\
\text { Medical Center, USA }\end{array}$ & In infancy & NS & 55 hours & 7,11 & NS & NS \\
\hline$\overline{\text { Zinner"w21 }}$ & CS & $\begin{array}{l}\text { Born at Boston City Hospital } \\
\text { or Womens and Infants } \\
\text { Hospital, Rhode Island, USA }\end{array}$ & In infancy & NS & $1-6$ days & 154,264 & NS & NS \\
\hline$\overline{\text { Dillon }}{ }^{w 7} \dagger$ & CS & $\begin{array}{l}\text { Born at Queen Charlotte's } \\
\text { Maternity Hospital, London, } \\
\text { UK }\end{array}$ & In infancy & NS & 6 days & 14,6 & $\begin{array}{l}\text { Standard formula } \\
\text { including Baby } \\
\text { Milk Plus, Cow } \\
\text { and Gate }\end{array}$ & NS \\
\hline Bernstein $^{w 1}$ & CS & $\begin{array}{l}\text { Born in Johannesburg } \\
\text { Hospital, South Africa }\end{array}$ & In infancy & $1987-8$ & 6 weeks & 43,39 & $\begin{array}{l}\text { Standard formula } \\
\text { (>10 mmol/l } \\
\text { sodium content) }\end{array}$ & $\begin{array}{l}\text { Yes, exclusively } \\
\text { breast fed or formula } \\
\text { fed }\end{array}$ \\
\hline Schachter ${ }^{w 17}$ & C & $\begin{array}{l}\text { Born at a large obstetric and } \\
\text { gynaecological hospital, USA }\end{array}$ & $\begin{array}{l}\text { Maternal records in } \\
\text { infancy }\end{array}$ & NS & 6 months & 30,141 & Not known & NS \\
\hline Pomeranz $^{\text {w14 }}$ & C & $\begin{array}{l}\text { Born in Meir General } \\
\text { Hospital, Israel }\end{array}$ & In infancy & NS & 6 months & 7,31 & $\begin{array}{l}\text { Standard formula } \\
\text { diluted with } \\
\text { mineral water or } \\
\text { tap water }\end{array}$ & NS \\
\hline Boulton"w2 & CS & $\begin{array}{l}\text { Born in Queen Victoria's } \\
\text { Hospital, seen at Adelaide } \\
\text { Children's Hospital, Australia }\end{array}$ & In infancy & $1976-9$ & 1 year & 14,47 & $\begin{array}{l}\text { Formula feed } \\
\text { including cow } \\
\text { milk, lactogen, } \\
\text { proprietary } \\
\text { simulated breast } \\
\text { milk. }\end{array}$ & $\begin{array}{l}\text { Unknown, breast fed } \\
\text { at 3-12 months, } \\
\text { artificially fed from } \\
<3 \text { months }\end{array}$ \\
\hline Zeman ${ }^{\text {w20 }}$ & CS & $\begin{array}{l}\text { Born at Czech Children's } \\
\text { Hospital, Prague, Czech } \\
\text { Republic }\end{array}$ & In infancy & NS & 8-26 months & 42,13 & $\begin{array}{l}\text { Formula feed } \\
\text { dried cows' milk }\end{array}$ & NS \\
\hline Baranowski ${ }^{13} \dagger$ & CS & $\begin{array}{l}\text { Studies of Children Activity } \\
\text { and Nutrition in Texas, USA }\end{array}$ & Parental interview & $1981-3$ & $3-4$ years & 60,185 & $\begin{array}{l}\text { Standard formula } \\
\text { including Enfamil, } \\
\text { Similac, SMA }\end{array}$ & $\begin{array}{l}\text { Yes, breast fed only } \\
\text { for }>3 \text { months or } \\
\text { bottle fed }\end{array}$ \\
\hline Whincup ${ }^{17} \dagger$ & CS & $\begin{array}{l}\text { Subjects recruited from } 9 \text { of } \\
24 \text { towns involved in the } \\
\text { British Regional Heart Study, } \\
\text { UK }\end{array}$ & $\begin{array}{l}\text { Parental } \\
\text { questionnaire at 5-8 } \\
\text { years }\end{array}$ & $1979-83$ & $5-8$ years & 1221,1787 & Standard formula & $\begin{array}{l}\text { Yes, exclusively } \\
\text { breast or bottle fed } \\
\text { for first } 3 \text { months }\end{array}$ \\
\hline Forsyth $^{12}$ & CS & $\begin{array}{l}\text { Recruited from four } \\
\text { European centres (Dundee, } \\
\text { Birmingham, UK; Leuven, } \\
\text { Belgium; Milan, Italy) }\end{array}$ & In infancy & 1992 & 5.8 years & 83,71 & Standard formula & NS \\
\hline Williams $^{w 19} \dagger$ & C & $\begin{array}{l}\text { Dunedin Multidisciplinary; } \\
\text { Health and Development } \\
\text { Study }\end{array}$ & $\begin{array}{l}\text { Parental } \\
\text { questionnaire at } 3 \\
\text { years }\end{array}$ & $1972-3$ & 7 and follow up at 18 & $\begin{array}{l}192,327 \\
197,319\end{array}$ & NS & $\begin{array}{l}\text { Yes, exclusively } \\
\text { breast fed (median } \\
28 \text { weeks) or bottle } \\
\text { fed }\end{array}$ \\
\hline Wilson ${ }^{14} \dagger$ & C & $\begin{array}{l}\text { Dundee infant feeding study, } \\
\text { Scotland }\end{array}$ & In infancy & $1983-6$ & $6-10$ years & 73,99 & Bottle fed & $\begin{array}{l}\text { Yes, exclusively } \\
\text { bottle fed or } \\
\text { exclusively breast fed } \\
\text { for } \geq 15 \text { weeks }\end{array}$ \\
\hline Lucas $^{18}$ & RCT & $\begin{array}{l}\text { Born at Norwich, Cambridge, } \\
\text { Sheffield, Ipswich, and } \\
\text { King's Lynn neonatal units, } \\
\text { UK }<1.85 \text { kg birth weight }\end{array}$ & In infancy & $1982-85$ & 7.5-8 Years & 66,60 & $\begin{array}{l}\text { Standard formula } \\
\text { (Farley's } \\
\text { Ostermilk) }\end{array}$ & $\begin{array}{l}\text { Yes, randomised to } \\
\text { breast milk or } \\
\text { preterm formula } \\
\text { within } 48 \text { hours of } \\
\text { birth }\end{array}$ \\
\hline Rona $^{\mathrm{w} 16} \dagger$ & CS & $\begin{array}{l}\text { National Study of Health and } \\
\text { Growth England } 1993 \\
\text { sample; England } 1994 \\
\text { sample; Scotland } 1994 \\
\text { sample }\end{array}$ & $\begin{array}{l}\text { All parental } \\
\text { questionnaire at } 9 \\
\text { years }\end{array}$ & $\begin{array}{l}1983-5 \\
1984-; \\
1983-6\end{array}$ & All 8-9 years & $\begin{array}{l}157,308 \\
213,318 \\
124,273\end{array}$ & All not known & $\begin{array}{l}\text { Yes, all exclusively } \\
\text { breast fed for } \geq 3 \\
\text { months or bottle fed }\end{array}$ \\
\hline $\begin{array}{l}\text { Esposito-Del } \\
\text { Puente }{ }^{\text {w9 }} \dagger\end{array}$ & CS & $\begin{array}{l}\text { Children from a primary } \\
\text { school in Naples, Italy }\end{array}$ & $\begin{array}{l}\text { Parental } \\
\text { questionnaire at 9-11 } \\
\text { years }\end{array}$ & $1980-2$ & 9-11 years & 43,17 & Standard formula & NS \\
\hline $\mathrm{BCS} 70^{\mathrm{w} 3} \dagger$ & C & $\begin{array}{l}\text { National Child Development } \\
\text { Study and British Cohort } \\
\text { Study } 1970\end{array}$ & $\begin{array}{l}\text { Parental } \\
\text { questionnaire at } 5 \\
\text { years }\end{array}$ & 1970 & 10 years & 951,5133 & Unknown & $\begin{array}{l}\text { No, breast fed partly } \\
\text { or wholly for } \geq 3 \\
\text { months or not breast } \\
\text { fed }\end{array}$ \\
\hline $\begin{array}{l}\text { Ten Towns Heart } \\
\text { Health Study }{ }^{19} \dagger\end{array}$ & Mixed C & $\begin{array}{l}\text { Ten Towns Heart Health } \\
\text { Study, UK }\end{array}$ & $\begin{array}{l}\text { Parental } \\
\text { questionnaire at 5-7 } \\
\text { years }\end{array}$ & $1982-6$ & 13-16 (mean 15) years & 980,951 & Standard formula & $\begin{array}{l}\text { Yes, exclusively } \\
\text { breast or bottle fed } \\
\text { for first } 3 \text { months }\end{array}$ \\
\hline Taittonen $^{15}$ & C & $\begin{array}{l}\text { Part of Cardiovascular Risk } \\
\text { Study in Finns }\end{array}$ & $\begin{array}{l}\text { Parental } \\
\text { questionnaire }\end{array}$ & $1962-74$ & 17 years & 760,150 & NS & $\begin{array}{l}\text { Unknown, exclusive } \\
\text { breast feeding status } \\
\text { not given, breast } \\
\text { feeding }>3 \text { months }\end{array}$ \\
\hline Kolacek ${ }^{\text {w11 }}$ & C & $\begin{array}{l}\text { Born in three locations in } \\
\text { Croatia }\end{array}$ & In infancy & $1968-9$ & $18-23$ years & 199,78 & $\begin{array}{l}27 \% \text { bottle fed, } \\
73 \% \text { fed diluted } \\
\text { cows' milk }\end{array}$ & $\begin{array}{l}\text { Yes, exclusively } \\
\text { breast or bottle fed } \\
\text { for first } 3 \text { months }\end{array}$ \\
\hline
\end{tabular}




\begin{tabular}{|c|c|c|c|c|c|c|c|c|}
\hline Study & Design & Source & $\begin{array}{l}\text { Source of } \\
\text { information } \\
\text { on feeding }\end{array}$ & $\begin{array}{l}\text { Year } \\
\text { born }\end{array}$ & $\begin{array}{l}\text { Age at } \\
\text { blood pressure } \\
\text { measurement }\end{array}$ & $\begin{array}{l}\text { No breast } \\
\text { fed, } \\
\text { No bottle fed }\end{array}$ & $\begin{array}{l}\text { Formula type, } \\
\text { comments }\end{array}$ & $\begin{array}{l}\text { Exclusive feeding } \\
\text { groups }\end{array}$ \\
\hline Leeson $^{\text {w12 }}$ & CS & $\begin{array}{l}\text { Born in Cambridge maternity } \\
\text { hospital, UK }\end{array}$ & Maternal recall & $1969-75$ & $20-28$ years & 149,182 & NS & $\begin{array}{l}\text { Yes, formula feeding } \\
\text { only or breast } \\
\text { feeding, but } \\
\text { minimum duration of } \\
\text { feeding not given }\end{array}$ \\
\hline Ravelliw15 & C & $\begin{array}{l}\text { Members of Dutch Famine } \\
\text { Birth Cohort }\end{array}$ & $\begin{array}{l}\text { Postnatal medical } \\
\text { records }\end{array}$ & $1943-7$ & $48-53$ years & 520,105 & $\begin{array}{l}\text { Diluted cows' } \\
\text { milk, added } \\
\text { sugar, buttermilk, } \\
\text { rice flour }\end{array}$ & $\begin{array}{l}\text { No, bottle fed and } \\
\text { partly breast fed, } \\
\text { feeding status } \\
\text { recorded } 10.4 \text { days } \\
\text { after birth }\end{array}$ \\
\hline Wadsworth ${ }^{w 18} \dagger$ & C & $\begin{array}{l}\text { Medical Research Council } \\
\text { National Survey of Health } \\
\text { and Development }\end{array}$ & In infancy & 1946 & 53 years & 1507,628 & NS & $\begin{array}{l}\text { Exclusively breast fed } \\
\text { for } \geq 3 \text { months or not } \\
\text { breast fed }\end{array}$ \\
\hline Fall $^{w 10} \dagger$ & C & $\begin{array}{l}\text { Adults born in Hertfordshire, } \\
\text { UK }\end{array}$ & Birth records & $1920-30$ & 59-71 years & 862,68 & $\begin{array}{l}\text { Probably cows' } \\
\text { milk }\end{array}$ & $\begin{array}{l}\text { Yes, exclusively } \\
\text { breast or bottle fed }\end{array}$ \\
\hline \multicolumn{6}{|c|}{$\begin{array}{l}\text { Overall pooled estimate based on random effects model due to heterogeneity between } 26 \text { estimates of SBP }(P<0.001), 23 \text { estimates } \\
\text { of DBP }(P=0.008)\end{array}$} & 8471,11292 & & \\
\hline
\end{tabular}

\section{Table 1(continued)}

\begin{tabular}{|c|c|c|c|c|c|}
\hline \multirow[b]{2}{*}{ Study (continued) } & \multirow[b]{2}{*}{ Type of sphygmomanometer, No of measures } & \multicolumn{2}{|c|}{ Mean (SE) systolic blood pressure (mm Hg) } & \multicolumn{2}{|c|}{ Diastolic blood pressure $(\mathrm{mm} \mathrm{Hg})$} \\
\hline & & Unadjusted & Adjusted & Unadjusted & Adjusted \\
\hline Cohen $^{\text {w/4 }}$ & Automated Dinamap (Critikon; Tampa, FL, USA), 3 & $2.40(3.48)$ & - & $-2.40(3.25)$ & - \\
\hline Zinner ${ }^{w 21}$ & $\begin{array}{l}\text { Automated Roche Arterisonde ultrasonic (Roche } \\
\text { Medical Electronics; Cranbury, NJ, USA), NS }\end{array}$ & $0.00(0.94)$ & - & $-0.70(0.92)$ & - \\
\hline Dillon ${ }^{w 7} \dagger$ & $\begin{array}{l}\text { Automated Doppler ultrasound system (Parkes) } \\
\text { and manual, NS }\end{array}$ & $4.11(5.13) \ddagger$ & - & $2.17(3.01) \ddagger$ & - \\
\hline Bernstein $^{w 1}$ & Unknown Doppler method, NS & $-4.10(2.00)$ & - & - & - \\
\hline Schachter ${ }^{\text {w17 }}$ & Automated Arteriosonde (Roche), 6 & $0.50(1.67)$ & - & $0.40(1.37)$ & - \\
\hline Pomeranz $^{\text {w14 }}$ & $\begin{array}{l}\text { Automated Dinamap } 8100 \text { vital signs (Critikon; } \\
\text { USA), NS }\end{array}$ & $-6.10(2.45)$ & - & $-7.30(3.20)$ & - \\
\hline Boulton $^{w 2}$ & Manual standard mercury, NS & $-4.11(3.12) \dagger \dagger$ & - & - & - \\
\hline Zeman $^{\text {w20 }}$ & Automated AVM 4, NS & $-4.91(2.10)$ & - & $0.33(0.90)$ & - \\
\hline Baranowski ${ }^{13} \dagger$ & $\begin{array}{l}\text { Automated Dinamap } 845 \text { XT/XT-IEC adult and } \\
\text { paediatric vital signs monitor (Critikon; USA), } 4\end{array}$ & $-2.17(1.61) \S$ & $-2.52(1.60)$ & $0.48(1.42) \S$ & $0.24(1.44)$ \\
\hline Whincup ${ }^{17} \dagger$ & Automated Dinamap 1846 SX P (Critikon; USA), 3 & $-0.20(0.33) \Uparrow$ & $-0.51(0.31)$ & $-0.26(0.23) \Uparrow$ & $-0.44(0.23)$ \\
\hline Forsyth $^{12}$ & $\begin{array}{l}\text { Automated Omron } 711 \text { (Omron Healthcare; } \\
\text { Hamburg, Germany), } 3\end{array}$ & $-2.2(1.6)$ & - & $-3.4(1.4)$ & - \\
\hline Williams ${ }^{\mathrm{w} 19} \dagger$ & $\begin{array}{l}\text { Manual mercury (London School of Hygiene, UK), } \\
2-3\end{array}$ & $\begin{array}{c}-0.50(0.66) \text { 凡, }-2.63 \\
(1.34) \text { 凡 }\end{array}$ & $\begin{array}{c}-0.84(0.64),-2.89 \\
(1.47)\end{array}$ & $\begin{array}{c}-0.70(0.61) \Re,-2.66 \\
(1.36) \Re\end{array}$ & $\begin{array}{c}-0.93(0.60),-2.78 \\
(1.42)\end{array}$ \\
\hline Wilson $^{14} \dagger$ & $\begin{array}{l}\text { Manual Hawksley Random Zero (Hawksley; } \\
\text { Lancing, Sussex, UK), NS }\end{array}$ & $-2.84(1.40) \uparrow$ & $-2.34(1.19)$ & $-1.79(1.23) \uparrow$ & $-1.49(1.23)$ \\
\hline Lucas $^{18}$ & $\begin{array}{l}\text { Automated Accutor Datascope (Datascope; } \\
\text { Paramus, NJ, USA) and manual, } 2\end{array}$ & $0.10(1.55)$ & - & $-0.70(1.32)$ & - \\
\hline $\operatorname{Rona}^{\text {w16 }} \dagger$ & Automated Dinamap 1846 (Critikon; USA), 3 & $\begin{array}{c}1.46(0.84) \text { ף; }-2.75 \\
(0.86) \text { ๆ; }-1.29(1.01) \text { ๆ }\end{array}$ & $\begin{array}{c}1.47(0.81) ;-2.58(0.80) ; \\
-1.57(1.01)\end{array}$ & 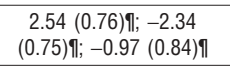 & $\begin{array}{c}2.57(0.75) ;-2.27(0.73) ; \\
-1.2(0.83)\end{array}$ \\
\hline Esposito-Del Puente ${ }^{\text {w9 }} \dagger$ & NS, NS & $-4.13(2.86) \uparrow$ & $-5(2.86)$ & $0.11(0.86)$ ף & $0.04(0.85)$ \\
\hline $\mathrm{BCS}^{2 \mathrm{w} 3} \dagger$ & Manual, 1 & $-0.16(0.38) \ddagger$ & $-0.50(0.38)$ & $-0.21(0.33) \ddagger$ & $-0.41(0.33)$ \\
\hline $\begin{array}{l}\text { Ten Towns Heart Health } \\
\text { Study }^{19} \dagger\end{array}$ & $\begin{array}{l}\text { Automated Dinamap } 1846 S X \text { oscillometric blood } \\
\text { pressure recorder (Critikon; USA), } 2\end{array}$ & $0.32(0.64)^{* *}$ & $0.24(0.59)$ & $-0.17(0.35)^{\star \star}$ & $-0.22(0.35)$ \\
\hline Taittonen $^{15}$ & Manual Hawksley Random Zero (Hawksley; UK), NS & $-5.48(1.14) \dagger \dagger$ & - & - & - \\
\hline Kolacek $^{\text {w11 }}$ & Manual Hawksley Random Zero (Hawksley; UK), 2 & $0.00(1.96)$ & - & $0.75(1.40)$ & - \\
\hline Leeson $^{\text {w12 }}$ & NS, NS & $0.00(1.55)$ & - & $-1.00(0.94)$ & \\
\hline Ravelliw15 $^{\text {w15 }}$ & $\begin{array}{l}\text { Automated Profilomat auscultatory device } \\
\text { (Diestronic Medical Systems Ag; Bugdorf, } \\
\text { Switzerland), } 4\end{array}$ & $0.20(1.65) \neq \ddagger$ & - & $0.90(1.08) \neq \ddagger$ & - \\
\hline Wadsworth ${ }^{\mathrm{w} 18} \dagger$ & $\begin{array}{l}\text { Automated Omron HEM-705CP (Omron; Tokyo, } \\
\text { Japan), } 1\end{array}$ & $-0.94(0.97)$ ף & $-0.57(0.97)$ & $-0.32(0.57)$ ף & $-0.05(0.57)$ \\
\hline Fallw10 $†$ & Automated Dinamap (Critikon; USA), 2 & $-2.64(2.88) \Uparrow$ & $-2.76(2.78)$ & $-0.96(1.38) \uparrow$ & $-1.00(1.35)$ \\
\hline $\begin{array}{l}\text { Overall pooled estimate } b \\
\text { between } 26 \text { estimates }\end{array}$ & $\begin{array}{l}\text { ed on random effects model due to heterogeneity } \\
\text { SBP }(P<0.001), 23 \text { estimates of } D B P(P=0.008)\end{array}$ & $\begin{array}{c}-1.10 \\
(95 \% \mathrm{Cl}-1.78 \text { to }-0.42) \\
\mathrm{P}=0.001\end{array}$ & $\begin{array}{c}-0.79 \\
(95 \% \mathrm{Cl}-1.42 \text { to }-0.16) \\
\mathrm{P}=0.014\end{array}$ & $\begin{array}{c}\quad-0.36 \\
(95 \% \mathrm{Cl}-0.79 \text { to } 0.08) \\
\mathrm{P}=0.111\end{array}$ & $\begin{array}{c}-0.39 \\
(95 \% \mathrm{Cl}-0.90 \text { to } 0.13) \\
\mathrm{P}=0.141\end{array}$ \\
\hline
\end{tabular}

$\mathrm{CS}=$ cross sectional; $\mathrm{C}=$ cohort; $\mathrm{RCT}=$ randomised controlled trial; $\mathrm{SBP}=$ systolic blood pressure; $\mathrm{DBP}=$ diastolic blood pressure; NS=not stated. *Additionally adjusted for height and body mass index. †Studies contributing previously unpublished data. łAdjusted for sex. §Adjusted for age, sex, and ethnicity. ๆAdjusted for age and sex. ${ }^{\star \star}$ Adjusted for age, town, ethnicity, sex, observer, and cuff size. ††Weighted average of male and female values. ¥¥Total sample standard deviation used for breast and bottle fed groups.

was not possible to distinguish formally on quality of blood pressure measurement (nearly all studies used automated machines) or on response rates, which were not provided for many studies.

\section{Results}

From 24 studies we obtained 26 mean differences in systolic blood pressure and 23 in diastolic blood pressure (table 1). All 
Table 2 Random effects meta-analyses by subgroup (study size, age group, and of those born before and after 1980)

\begin{tabular}{|c|c|c|c|c|c|c|c|}
\hline \multirow[b]{2}{*}{ Subgroup analysis } & \multicolumn{2}{|c|}{$\begin{array}{l}\text { No of estimates of } \\
\text { blood pressure }\end{array}$} & \multirow[b]{2}{*}{$\begin{array}{c}\text { No breast fed, No } \\
\text { bottle fed }\end{array}$} & \multicolumn{4}{|c|}{ Mean $(95 \% \mathrm{Cl})$ difference in blood pressure } \\
\hline & $\begin{array}{l}\text { Systolic blood } \\
\text { pressure }\end{array}$ & $\begin{array}{l}\text { Diastolic } \\
\text { blood } \\
\text { pressure }\end{array}$ & & Systolic blood pressure & $P$ value & Diastolic blood pressure & $P$ value \\
\hline \multicolumn{8}{|l|}{ By study size } \\
\hline$<300$ participants & 13 & 11 & 681,798 & $-2.05(-3.30$ to -0.80$)$ & 0.001 & $-0.53(-1.52$ to 0.46$)$ & 0.295 \\
\hline 300-1000 participants & 9 & 8 & 3131,1995 & $-1.13(-2.53$ to 0.27$)$ & 0.112 & $-0.40(-1.52$ to 0.72$)$ & 0.485 \\
\hline$>1000$ participants & 4 & 4 & 4659,8499 & $-0.16(-0.60$ to 0.28$)$ & 0.480 & $-0.24(-0.55$ to 0.08$)$ & 0.142 \\
\hline \multicolumn{8}{|l|}{ By age group } \\
\hline Infants ( $\leq 1$ years) & 7 & 5 & 269,539 & $-1.43(-3.69$ to 0.84$)$ & 0.217 & $-0.83(-2.88$ to 1.22$)$ & 0.427 \\
\hline Children (>1 to 16 years) & 13 & 13 & 4205,9542 & $-0.78(-1.48$ to -0.07$)$ & 0.031 & $-0.37(-0.93$ to 0.18$)$ & 0.188 \\
\hline Adults ( $\geq 17$ years) & 7 & 6 & 4194,1530 & $-1.75(-3.51$ to 0.02$)$ & 0.052 & $-0.45(-1.27$ to 0.37$)$ & 0.284 \\
\hline \multicolumn{8}{|l|}{ By year of birth } \\
\hline Born before 1980 & 13 & 11 & 5394,7142 & $-1.07(-2.12$ to -0.02$)$ & 0.045 & $-0.25(-0.68$ to 0.18$)$ & 0.256 \\
\hline Born after 1980 & 13 & 12 & 3077,4150 & $-1.20(-2.20$ to -0.20$)$ & 0.018 & $-0.61(-1.42$ to 0.20$)$ & 0.138 \\
\hline
\end{tabular}

were observational studies, except for one randomised controlled trial in preterm infants. ${ }^{18}$ Non-randomised comparisons of breastfed and bottle fed infants were obtained from another randomised controlled trial. ${ }^{12}$ Eight observations on systolic blood pressure were in infants, 12 were in children, and six were in adults (fig 1). In a random effects model including all studies, mean systolic blood pressure was lower in breastfed participants than in bottle fed participants $(-1.10 \mathrm{~mm} \mathrm{Hg}, 95 \%$ confidence interval -1.78 to $-0.42 \mathrm{~mm} \mathrm{Hg}$ ). We found noticeable heterogeneity between studies $\left(\chi^{2}=59.4, \mathrm{df}=25, \mathrm{P}<0.001\right)$. No consistent difference was, however, found either between the three age groups $(\mathrm{P}=0.601)$ or between studies in participants born before and after $1980(\mathrm{P}=0.832$, table 2$)$.

Studies that reported noticeable and statistically significant differences in blood pressure between feeding groups were mostly small, raising the possibility of publication bias (see fig 1). Evidence of such bias was provided by a funnel plot (fig 2). The Egger test was significant $(\mathrm{P}=0.033)$ for publication bias but not the Begg test $(\mathrm{P}=0.186)$. The estimate of effect size decreased with increasing study size: $-2.05 \mathrm{~mm} \mathrm{Hg}$ in the 13 studies with fewer than 300 participants, $-1.13 \mathrm{~mm} \mathrm{Hg}$ in the seven studies (nine observations) with 300 to 1000 participants, and -0.16 $\mathrm{mm} \mathrm{Hg}$ in the four studies with more than 1000 participants (test for trend between groups $\mathrm{P}=0.046)$. However, a test for trend with study size treated as a continuous variable, was not significant $(\mathrm{P}=0.209)$. In 10 studies (12 observations) in which we were able to examine the effect of adjustment for current body size, the difference was similar both before and after adjustment. The effect size seemed greatest in 16 studies that had reported on the association between infant feeding method and systolic blood pressure $(-1.67 \mathrm{~mm} \mathrm{Hg},-2.80$ to $-0.53 \mathrm{~mm} \mathrm{Hg})$ than in 10 studies that did not $(-0.54 \mathrm{~mm} \mathrm{Hg},-1.36$ to 0.28 $\mathrm{mm} \mathrm{Hg})$. This difference was not, however, significant $(\mathrm{P}=0.194)$.

Mean diastolic blood pressure showed no noticeable difference between breastfed and bottle fed groups (fig 3 and table 2). In a random effects model, breastfed participants had similar mean diastolic blood pressure to bottle fed participants $(-0.36$ $\mathrm{mm} \mathrm{Hg},-0.79$ to $0.08 \mathrm{~mm} \mathrm{Hg}$ ). Significant heterogeneity was found between studies $\left(\chi^{2}=38.9, \mathrm{df}=22, \mathrm{P}=0.014\right)$, although there was no consistent difference between age groups $(\mathrm{P}=0.96)$ and no difference between participants born before and after $1980(\mathrm{P}=0.80)$. This difference was unaltered when the analysis was restricted to studies that adjusted for current body size. We found no evidence of publication or inclusion bias, and the results were similar for studies of different sizes.

The difference in blood pressure between feeding groups was unaltered by infant feeding status being recorded in infancy $(\mathrm{n}=15)$ or by parent administered questionnaire $(\mathrm{n}=11)$ at least three years after birth $(\mathrm{P}=0.593){ }^{13}{ }^{1319}$ No important effects were found on the results for either systolic or diastolic blood pressure after the exclusion of 12 studies where feeding was not exclusive or could not be confirmed. ${ }^{12} 15 \mathrm{w} 2-\mathrm{w} 4 \mathrm{w} 7 \mathrm{w} 9 \mathrm{w} 14 \mathrm{w} 15 \mathrm{w} 17 \mathrm{w} 20 \mathrm{w} 21$

\section{Discussion}

Our systematic review found that publication bias may partly explain the lower mean systolic blood pressure observed in participants that had been breast fed in infancy, with large studies showing little difference. Studies with more than 1000 participants, which are less subject to publication bias, reliably excluded a mean difference greater than $0.6 \mathrm{~mm} \mathrm{Hg}$, suggesting an effect of little clinical or public health importance. Feeding in infancy was not significantly associated with diastolic blood pressure.

Even if publication bias is discounted, the overall difference in systolic blood pressure of $1.1 \mathrm{~mm} \mathrm{Hg}$ is modest. The relatively weak association between infant feeding and systolic blood pressure is not likely to reflect imprecise ascertainment of early feeding practices, as most were documented either during infancy from health records or from questionnaires administered to parents, the accuracy of which has shown to be valid up to 20 years after birth. ${ }^{24}$ In addition, the difference in blood pressure between feeding groups was similar in studies that recorded infant feeding status in infancy to those based on a parental questionnaire later in life.

Random allocation of infants to breast feeding or bottle feeding has been regarded as inappropriate, except in the special

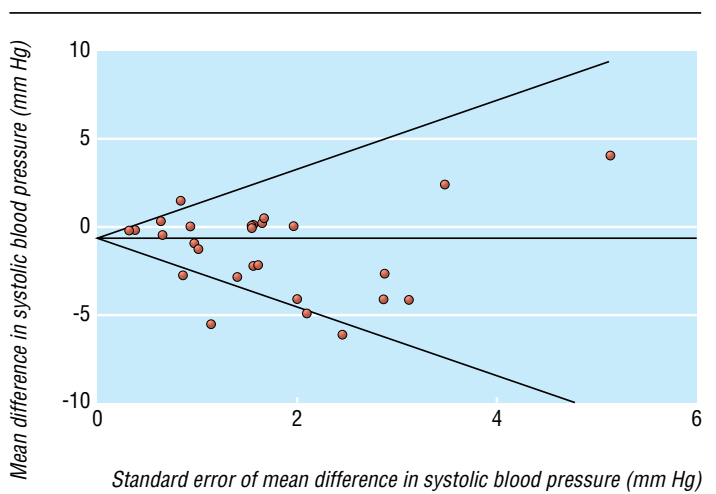

Fig 2 Begg's funnel plot (pseudo 95\% confidence limits) showing mean difference in systolic blood pressure by standard error of mean difference 


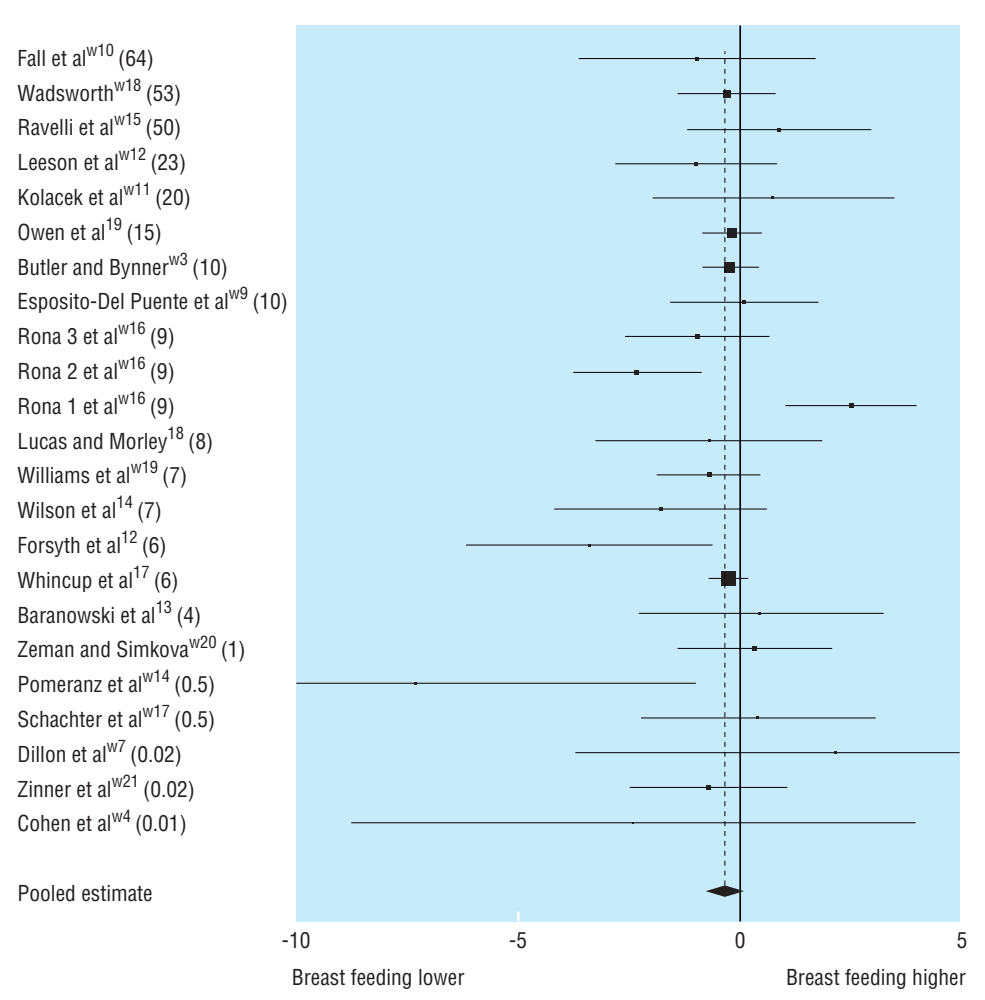

Mean difference in diastolic blood pressure $(\mathrm{mm} \mathrm{Hg})$

Fig 3 Mean differences in diastolic blood pressure (95\% confidence intervals) between breastfed and bottle fed participants. Box area proportional to inverse of variance, with horizontal lines showing $95 \%$ confidence intervals. y axis in ascending order of mean age (years) at which blood pressure was measured. Dashed vertical line and diamond ( $95 \%$ confidence interval) is pooled estimate based on a random effects model

circumstances of preterm birth, in which one randomised trial has been carried out. ${ }^{18}$ That trial showed no marked difference in blood pressure between 8 year old children who were breast fed or bottle fed as infants. ${ }^{18}$ A follow up examination showing noticeably higher mean blood pressures among bottle fed infants was based on only a quarter of the study population; at such low follow up rates the validity of the comparisons cannot be assumed, and the degree of control for the original study centre has been questioned. ${ }^{25}$ The other studies in this review were observational, so that there is a possibility of confounding, particularly by social factors, current body size, and diet in later life. However, since bottle feeding tends to be related to lower social class, a greater tendency to obesity, and a less healthy diet in later life, all of which are likely to be related to higher mean blood pressure, any confounding effects are likely to have exaggerated, rather than reduced, the extent to which mean blood pressure levels are higher among participants that were bottle fed. ${ }^{26} 27$

Although our review effectively excludes any important overall lowering effect of breast feeding on blood pressure, it is possible that prolonged breast feeding has a protective effect. ${ }^{15}$ This issue requires further systematic examination.

We were unable to examine whether infants fed formula milk supplemented with long chain polyunsaturated fatty acids had lower blood pressure in childhood compared with infants fed standard formulas. ${ }^{12}$ The results do not, however, suggest that naturally occurring long chain polyunsaturated fatty acids in breast milk have an appreciable effect on blood pressure. The results from large studies effectively exclude the effect size observed in the recently reported small trial of supplementation with long chain polyunsaturated fatty acids, although the confidence intervals around that estimate were wide. ${ }^{12}$ The absence of marked differences in blood pressure between infant feeding groups (either before or after 1980) are also of interest because of the higher sodium content of formula milk up to $1980 .{ }^{5}{ }^{6}$ In the $1970 \mathrm{~s}$, the average sodium intake of formula fed infants at one month of age in most of the populations studied here would have been around $20 \mathrm{mmol}$ a day higher than that of infants who were breast fed. ${ }^{5}$ However, the interpretation of the results depends on the strength of association between sodium intake and blood pressure assumed. Using the large effect estimates derived from a randomised controlled trial of salt restriction in neonates, a $20 \mathrm{mmol}$ a day difference might be expected to produce a difference in systolic blood pressure in the order of 5-6 $\mathrm{mm} \mathrm{Hg}$ between infants that were breast fed or bottle fed-a difference that is easy to exclude among the infants in four studies before 1980 (data not presented). ${ }^{7}$ However, the more conservative effect estimates from systematic reviews of sodium reduction trials in adults would be consistent with differences between bottle fed and breastfed infants of 1-2 mm Hg, which would be considerably more difficult to exclude with confidence. ${ }^{28}{ }^{29}$ The absence of any appreciable change in the size of this difference between the 1970s and 1990s, particularly in the largest studies, suggests that the effects of changing sodium intake in infancy on subsequent blood pressure have been modest.

\section{Conclusions}

The lower levels of systolic blood pressure associated with breast feeding in infancy identified in our review were observed mainly 


\section{What is already known on this topic}

Early studies suggested that breast feeding in infancy may protect against high blood pressure in later life

Many studies examining this effect have been small, raising the possibility of publication bias

\section{What this study adds}

Selective publication of small studies shows that breast feeding reduces blood pressure in later life

The effect in larger studies seems to be small and of limited clinical or public health importance

in small studies; with little difference shown in studies of 1000 participants or more. The association of breast feeding and lower blood pressure may well be partly explained by publication bias. Hence the results of small studies showing large differences in blood pressure should be treated cautiously. Our analysis suggests that any effect of breast feeding on blood pressure is modest and of limited clinical or public health importance. However, blood pressure is not the only relevant outcome; the case for breast feeding rests on a combination of short and long term benefits, including improved neural and psychosocial development, potential protection against obesity and allergic disease, and lower blood cholesterol levels in later life. ${ }^{19} 26$ 30-33

We thank the following investigators for providing data: $\mathrm{R}$ Rona and $\mathrm{S}$ Chinn (Guy's, King's, and St Thomas's School of Medicine, London), S Kolacek (Children's Hospital Zagreb, Croatia), T Baranowski and F Bradford (Children's Nutrition Research Center, Baylor College of Medicine, USA), J S Forsyth (Ninewells Hospital and Medical School, Dundee, Scotland), C Fall (Medical Research Council Environmental Epidemiology Unit, University of Southampton), A Esposito-Del Puente (Dipartimento di Medicina Clinica e Sperimentale, Università degli Studi di Napoli Federico II, Italy), M J Dillon (Institute of Child Health, London), S Williams (Dunedin School of Medicine, University of Otago, New Zealand), and M Wadsworth (Department of Epidemiology and Public Health, University College London). We acknowledge the original data creators, depositors, copyright holders, the funders of the Data Collections, and the UK Data Archive (University of Essex, Colchester, UK) for use of data from the 1970 British Cohort Study. They bear no responsibility for the analysis or interpretation of this data.

Contributors: CGO, PHW, and DGC had the idea for this paper: CGO extracted the data and carried out the statistical analysis with the support of JAG. CGO drafted the paper to which all authors contributed; he will act as guarantor for the paper.

Funding: The third phase of the ten towns heart health study was supported by the Wellcome Trust (grant No $051187 / \mathrm{Z} / 97 / \mathrm{A})$.

Competing interests: None declared.

Ethical approval: Not required.

1 Prospective Studies Collaboration. Age-specific relevance of usual blood pressure to vascular mortality: a meta-analysis of individual data for one million adults in 61 prospective studies. Lancet 2002;360:1903-13.

2 Lever AF, Harrap SB. Essential hypertension: a disorder of growth with origins in childhood? J Hypertension 1992;10:101-20.

3 Barker DJ. Mothers, babies and health in later life. London: Churchill Livingstone, 1998.

4 Lucas A. Programming by early nutrition in man. In: Bock GR, Whelan J, eds. Childhood Lucas A. Programming by early nutrition in man.
environment and adult disease. Wiley, 1991:38-55.

5 Oppe TE. Present-day practice in infant feeding. Report of a working party of the Panel on Child Nutrition, Committee on Medical Aspects of Food Policy. London: HMSO, 1974.
6 Oppe TE. Artificial feeds for the young infant. Report of the Working Party on the Composition of Foods for Infants and Young Children, Committee on Medical Aspects of Food Policy. London: HMSO, 1980

7 Hofman A, Hazebroek A, Valkenburg HA. A randomized trial of sodium intake and blood pressure in newborn infants. JAMA 1983;250:370-3.

8 Geleijnse JM, Hofman A, Witteman JC, Hazebroek AA, Valkenburg HA, Grobbee DE. Long-term effects of neonatal sodium restriction on blood pressure. Hypertension 1997;29:913-7.

9 Cunnane SC, Francescutti V, Brenna JT, Crawford MA. Breast-fed infants achieve a higher rate of brain and whole body docosahexaenoate accumulation than formula-fed infants not consuming dietary docosahexaenoate. Lipids 2000;35:105-11.

10 Engler MM, Engler MB, Kroetz DL, Boswell KD, Neeley E, Krassner SM. The effects of a diet rich in docosahexaenoic acid on organ and vascular fatty acid composition in spontaneously hypertensive rats. Prostaglandins Leukot Essent Fatty Acids 1999;61:28995.

11 Mori TA, Bao DQ, Burke V, Puddey IB, Beilin LJ. Docosahexaenoic acid but not eicosapentaenoic acid lowers ambulatory blood pressure and heart rate in humans. Hypertension 1999;34:253-60.

12 Forsyth JS, Willatts P, Agostoni C, Bissenden J, Casaer P, Boehm G. Long chain polyunsaturated fatty acid supplementation in infant formula and blood pressure in later childhood: follow up of a randomised controlled trial. BMJ 2003;326:953.

13 Baranowski T, Bryan GT, Harrison JA, Rassin DK, Greaves KA, Baranowski JH. Height, infant-feeding practices and cardiovascular functioning among 3 or 4 year old children in three ethnic groups. J Clin Epidemiol 1992;45:513-8.

14 Wilson AC, Forsyth JS, Greene SA, Irvine L, Hau C, Howie PW. Relation of infant diet to childhood health: seven year follow up of cohort of children in Dundee infant feeding study. BMJ 1998;316:21-5.

15 Taittonen L, Nuutinen M, Turtinen J, Uhari M. Prenatal and postnatal factors in predicting later blood pressure among children: cardiovascular risk in young Finns. Pediatr Res 1996;40:627-32.

16 Singhal A, Cole TJ, Lucas A. Early nutrition in preterm infants and later blood pressure: two cohorts after randomised trials. Lancet 2001;357:413-9.

17 Whincup PH, Cook DG, Shaper AG. Early influences on blood pressure: a study of children aged 5-7 years. BMJ 1989;299:587-91.

18 Lucas A, Morley R. Does early nutrition in infants born before term programme later blood pressure? BMJ 1994;309:304-8.

19 Owen CG, Whincup PH, Odoki K, Gilg JA, Cook DG. Infant feeding and blood cholesterol: a study in adolescents and a systematic review. Pediatrics 2002;110:597-608.

terol: a study in adolescents and a systematic review. Pediatrics 2002;110:597-608. 88 .

21 Light RJ, Pillemar DB. Summing up: the science of reviewing research. Cambridge, MA: Harvard University Press, 1984.

22 Begg CB, Mazumdar M. Operating characteristics of a rank correlation test for publication bias. Biometrics 1994;50:1088-101.

23 Egger M, Davey Smith G, Schneider M, Minder C. Bias in meta-analysis detected by a simple, graphical test. $B M J$ 1997;315:629-34.

24 Kark JD, Troya G, Friedlander Y, Slater PE, Stein Y. Validity of maternal reporting of breast feeding history and the association with blood lipids in 17 year olds in Jerusalem. JEpidemiol Community Health 1984;38:218-25.

25 Roberts SB. Prevention of hypertension in adulthood by breastfeeding? Lancet Roberts SB. Pre
2001;357:406-7.

26 Gillman MW, Rifas-Shiman SL, Camargo CA Jr, Berkey CS, Frazier AL, Rockett HR, et al. Risk of overweight among adolescents who were breastfed as infants. JAMA 2001;285:2461-7.

27 Colhoun HM, Hemingway H, Poulter NR. Socio-economic status and blood pressure: an overview analysis. J Hum Hypertens 1998;12:91-110.

28 Cutler JA, Follmann D, Allender PS. Randomized trials of sodium reduction: an overview. Am J Clin Nutr 1997;65:643-51S.

29 Law MR, Frost CD, Wald NJ. By how much does dietary salt reduction lower blood pressure? III, Analysis of data from trials of salt reduction. BMJ 1991;302:819-24.

30 Makrides M, Neumann M, Simmer K, Pater J, Gibson R. Are long-chain Makrides M, Neumann M, Simmer K, Pater J, Gibson R. Are long-chain

31 Fergusson DM, Woodward LJ. Breast feeding and later psychosocial adjustment. Paediatr Perinat Epidemiol 1999;13:144-57.

32 Hediger ML, Overpeck MD, Kuczmarski RJ, Ruan WJ. Association between infant breastfeeding and overweight in young children. JAMA 2001;285:2453-60.

33 Lucas A, Brooke OG, Morley R, Cole TJ, Bamford MF. Early diet of preterm infants and development of allergic or atopic disease: randomised prospective study. $B M$ J 1990;300:837-40.

(Accepted 15 September 2003)

bmj.com 2003;327:1189

Department of Community Health Sciences, St George's Hospital Medical School, London SW17 ORE

Christopher G Owen epidemiologist

Peter H Whincup professor of cardiovascular epidemiology

Julie A Gilg statistician

Derek G Cook professor of epidemiology

Correspondence to: $\mathrm{C}$ G Owen

c.owen@sghms.ac.uk 